\title{
Histopathologic and clinicopathologic classifications of antineutrophil cytoplasmic antibody-associated glomerulonephritis: a validation study in a Korean cohort
}

\author{
Jeong-Hoon Lim ${ }^{1,2}$, Man-Hoon Han $^{3}$, Yong-Jin $\mathrm{Kim}^{3}$, Yena Jeon ${ }^{4}$, Hee-Yeon Jung ${ }^{1,5}$, Ji-Young Choi ${ }^{1,2}$, \\ Jang-Hee Cho ${ }^{1,5}$, Chan-Duck Kim ${ }^{1,5}$, Yong-Lim Kim ${ }^{1,5}$, Hajeong Lee ${ }^{6,7}$, Dong Ki Kim ${ }^{6,7}$, Kyung Chul Moon ${ }^{8}$, \\ Sun-Hee Park ${ }^{1,5}$ \\ ${ }^{1}$ Department of Internal Medicine, School of Medicine, Kyungpook National University, Daegu, Republic of Korea \\ ${ }^{2}$ Department of Internal Medicine, Kyungpook National University Chilgok Hospital, Daegu, Republic of Korea \\ ${ }^{3}$ Department of Pathology, School of Medicine, Kyungpook National University, Kyungpook National University Hospital, Daegu, Republic of \\ Korea \\ ${ }^{4}$ Department of Statistics, Kyungpook National University, Daegu, Republic of Korea \\ ${ }^{5}$ Department of Internal Medicine, Kyungpook National University Hospital, Daegu, Republic of Korea \\ ${ }^{6}$ Department of Internal Medicine, Seoul National University School of Medicine, Seoul, Republic of Korea \\ ${ }^{7}$ Department of Internal Medicine, Seoul National University Hospital, Seoul, Republic of Korea \\ ${ }^{8}$ Department of Pathology, Seoul National University College of Medicine, Seoul, Republic of Korea
}

Background: Antineutrophil cytoplasmic antibodies (ANCA)-associated glomerulonephritis (AAGN) is a common cause of rapidly progressive glomerulonephritis and requires prompt and proper immunosuppressive therapy to improve renal prognosis. This study aimed to evaluate the predictive value of two different classifications for renal outcomes in Korean AAGN patients.

Methods: Ninety-two patients who were diagnosed with AAGN at two tertiary hospitals between 2004 and 2018 were retrospectively analyzed retrospectively. The histopathologic classification according to glomerular pathology and the clinicopathologic classification according to normal glomeruli ratio, degree of interstitial fibrosis/tubular atrophy, and baseline renal function were evaluated using the Cox proportional hazards model.

Results: Forty-five patients (48.9\%) progressed to end-stage kidney disease (ESKD) during the observation period. The mean age was $61.0 \pm 15.3$ years, and most patients had myeloperoxidase-ANCA (93.5\%). In the histopathologic classification, the best renal survival occurred in the focal class, whereas the sclerotic class had the worst renal survival (sclerotic class vs. focal class; adjusted hazard ratio [aHR], 5.05; 95\% confidence interval $[\mathrm{Cl}], 1.32-19.31 ; p=0.018)$. The mixed class had intermediate renal outcomes (mixed class vs. focal class; aHR, 4.23; 95\% Cl, 1.23-14.58; $p=0.022$ ). In the clinicopathologic classification, the high-risk group had poor renal outcomes compared with the low-risk group (aHR, 6.56; 95\% Cl, 1.25-34.26; p = 0.026), but renal outcomes did not differ between the low- and medium-risk groups.

Received: October 8, 2020; Revised: November 11, 2020; Accepted: November 22, 2020

Editor: Beom Jin Lim, Yonsei University, Seoul. Republic of Korea

Correspondence: Sun-Hee Park

Department of Internal Medicine, School of Medicine, Kyungpook National University, Kyungpook National University Hospital, 130 Dongdeok-ro, Jung-gu, Daegu 41944, Republic of Korea. E-mail:sh-park@knu.ac.kr

ORCID: https://orcid.org/0000-0002-0953-3343

Copyright @ 2021 The Korean Society of Nephrology

(a) This is an Open Access article distributed under the terms of the Creative Commons Attribution Non-Commercial and No Derivatives License (http:// creativecommons.org/licenses/by-nc-nd/4.0/) which permits unrestricted non-commercial use, distribution of the material without any modifications, and reproduction in any medium, provided the original works properly cited. 
Conclusion: In Korean AAGN patients, histopathologic and clinicopathologic classifications had predictive value for renal outcomes, especially in the sclerotic class or the high-risk group with higher risk of progression to ESKD despite treatment.

Keywords: Antibodies, Antineutrophil cytoplasmic, Classification, Glomerulonephritis, Kidney failure, Chronic, Pathology

\section{Introduction}

Antineutrophil cytoplasmic antibodies (ANCA)-associated vasculitis (AAV) is characterized by necrotizing inflammation of small vessels and consists of a group of multisystemic diseases, such as microscopic polyangiitis, granulomatosis with polyangiitis, eosinophilic granulomatosis with polyangiitis, and renal limited vasculitis [1,2]. AAV commonly involves the kidneys and is a common cause of rapidly progressive glomerulonephritis [3]. Despite treatment, renal survival and patient survival are poor in patients with ANCA-associated glomerulonephritis (AAGN) $[4,5]$. Prompt diagnosis and immediate proper immunosuppressive therapy are necessary to prevent the progression of end-stage kidney disease (ESKD). However, immunosuppression can also cause an increased in short-term or long-term mortality, mainly by infection [6].

To prevent excessive immunosuppression and reduce complications, several studies have been conducted to find the histopathologic or clinical predictors for renal prognosis at the time of diagnosis. Various parameters, such as age, baseline renal function, percentage of normal/globally sclerotic glomeruli, and degree of interstitial fibrosis/tubular atrophy (IF/TA), have been identified as possible predictors $[4,5,7,8]$. However, they have limitations in predicting renal outcomes and have not been validated. Berden et al. [9] developed a simple histopathologic classification of AAGN divided into four classes (focal, crescentic, mixed, and sclerotic) according to glomeruli and crescent types. More recently, Brix et al. [10] accentuated the limitations of the histopathologic classification that reflects only glomerular findings. They developed a new classification according to the ANCA kidney risk scoring system that reflects not only histopathologic but also clinical findings.

Both classifications were developed using data from western AAV patients. Although there are Chinese and Japanese validation studies for histopathologic classification $[11,12]$, no studies have used a Korean cohort. Also, there are still no studies on the ANCA kidney risk score for Asian AAGN patients. Therefore, this study aimed to evaluate the predictive value of the histopathologic and clinicopathologic classifications for renal outcomes among Korean AAGN patients.

\section{Methods}

\section{Patients}

This retrospective cohort study analyzed all patients diagnosed with AAGN at two university-based tertiary hospitals (Kyungpook National University Hospital and Seoul National University Hospital) from March 2004 to March 2018. Patients were eligible for inclusion if they met the following: (1) the criteria of the Chapel Hill Consensus Conference definition for AAV [2], (2) were positive serology for ANCA, (3) renal biopsy consistent with AAGN and the specimen contained $\geq 10$ glomeruli $[5,9]$, and (4) were followed up for $\geq 1$ year. A total of 92 patients were included in this study. The study protocol was reviewed and approved by the Institutional Review Boards of Kyungpook National University Hospital (No. 2017-08-013-003) and Seoul National University Hospital (No. H1802-102-924). Informed consent was waived, as there was no infringement of the patients' health or privacy during the study.

\section{Data collection and definition}

Data on patient demographics, comorbid diseases, and laboratory findings were surveyed at the time of renal biopsy from electronic medical records. Renal function was measured again at 1 year after diagnosis. The estimated glomerular filtration rate (eGFR) was calculated using the Chronic Kidney Disease Epidemiology Collaboration (CKD-EPI) creatinine equation [13]. Information on the development of ESKD and the last follow-up date was also collected. The development of ESKD was defined as requiring kidney transplantation, permanent hemodialysis, or peritoneal dialysis. Hypertension was defined as systolic blood pressure of $\geq 140 \mathrm{mmHg}$ and/or diastolic blood 
pressure of $\geq 90 \mathrm{mmHg}$ or the use of antihypertensive medication. Diabetes was defined as the use of glucoselowering agents, random glucose of $\geq 200 \mathrm{mg} / \mathrm{dL}$, or hemoglobin Alc of $\geq 6.5 \%$.

Two renal pathologists (MHH and YJK) who were blinded to the patient information independently evaluated the renal specimens. The details of the evaluated histopathologic information are as follows: the number of total/normal/ crescentic (cellular or fibrous)/globally sclerotic glomeruli, histopathologic classification of AAGN using the algorithm developed by Berden et al. [9], and degree of IF/TA. The histopathologic classification was divided into four categories: focal class ( $\geq 50 \%$ normal glomeruli), crescentic class $(\geq 50 \%$ cellular crescentic glomeruli), sclerotic class $(\geq 50 \%$ globally sclerotic glomeruli), and mixed class $(<50 \%$ normal, cellular crescentic, and globally sclerotic glomeruli) [9]. The detailed information and histopathologic images are shown in our previous report [14]. The interobserver variations of the histopathologic classification and the clinicopathologic score were identified $(\kappa=0.89$ and $\kappa=$ 0.95 , respectively), and the discrepancies between the two observers were resolved by consensus meetings according to the recommendation [15]. The clinicopathologic classification was divided into three groups (low, medium, and high) according to the risk scores that consist of the histopathologic parameters (percentage of normal glomeruli: $>25 \%$, 0 points; $10 \%-25 \%$, 4 points; $<10 \%$, 6 points and degree of IF/TA: $\leq 25 \%, 0$ points; $>25 \%, 2$ points) and the laboratory variable (eGFR at the time of diagnosis: $>15 \mathrm{~mL} /$ $\min / 1.73 \mathrm{~m}^{2}, 0$ points; $\leq 15 \mathrm{~mL} / \mathrm{min} / 1.73 \mathrm{~m}^{2}, 3$ points) [10]. The risk groups were defined according to the sum scores: low-risk group, 0 points; medium-risk group, 2 to 7 points; and high-risk group, 8 to 11 points.

\section{Treatment}

Individual treatment information could not be retrieved in the data. The treatment was decided based on the general condition of the patient, comorbid diseases, and treatment protocols for AAGN $[16,17]$. Detailed information for general AAGN treatment has been described in a previous study [14]. In brief, high-dose intravenous methylprednisolone ( $500 \mathrm{mg} /$ day for 3 days) with pulse intravenous or daily oral cyclophosphamide was used for induction. The steroid was then tapered to $1 \mathrm{mg} / \mathrm{kg} /$ day oral prednisolone for 4 to 6 weeks. Low-dose oral prednisolone and oral azathioprine were used for maintenance immunosuppression. Rituximab was used as induction immunosuppressive therapy for 21 patients (22.8\%) since 2011.

\section{Statistical analysis}

Kolmogorov-Smirnov test was used to evaluate the normal distribution of variables. Continuous variables are presented as mean \pm standard deviation or median (interquartile range, IQR) according to the distribution. Categorical variables are presented as number (percentage). Student $t$ test or Mann-Whiney U test was used to compare the differences in continuous variables. Pearson chi-square test or Fisher exact test was used to compare the differences in categorical variables, as appropriate. Kaplan-Meier analysis was used to compare renal survival. A log-rank test was used to analyze the difference. Linear regression analysis was used to identify the associated factors for the 1-year change in eGFR. Cox proportional hazards regression analysis was performed to identify the predictors for renal survival. The statistic was used to evaluate the interobserver difference for the histopathologic classification. Statistical analyses were performed with IBM SPSS for Windows, version 22 (IBM Corp., Armonk, NY, USA). A p-value of $<0.05$ was considered statistically significant.

\section{Results}

\section{Baseline characteristics}

Among 92 AAGN patients, 45 (48.9\%) had developed ESKD during a median of 475 days of follow-up (Table 1). The mean age was $61.0 \pm 15.3$ years at the time of diagnosis. Patients with ESKD had a tendency to be older than those without ESKD (63.4 \pm 15.4 years vs. $57.1 \pm 15.6$ years; $p=$ 0.055). Hypertension was more common in the ESKD group than in the non-ESKD group $(46.7 \%$ vs. $21.3 \%$; $p=0.010)$. Baseline eGFR was significantly lower among ESKD patients $\left(8.6 \mathrm{~mL} / \mathrm{min} / 1.73 \mathrm{~m}^{2}\left[7.0-13.2 \mathrm{~mL} / \mathrm{min} / 1.73 \mathrm{~m}^{2}\right]\right.$ vs. 24.0 $\mathrm{mL} / \mathrm{min} / 1.73 \mathrm{~m}^{2}$ [12.9-40.2 mL/min/1.73 $\left.\left.\mathrm{m}^{2}\right] ; \mathrm{p}<0.001\right)$. Most patients had myeloperoxidase (MPO)-ANCA (86 of 92, 93.5\%), and the proportion was similar between the ESKD and non-ESKD groups.

For the histopathologic findings, the median number of 
Table 1. Baseline characteristics at the time of diagnosis

\begin{tabular}{|c|c|c|c|c|}
\hline Variable & Total & ESKD & Non-ESKD & p-value \\
\hline No. of patients & 92 & 45 & 47 & \\
\hline Age (yr) & $61.0 \pm 15.3$ & $63.4 \pm 15.4$ & $57.1 \pm 15.6$ & 0.055 \\
\hline \multicolumn{5}{|l|}{ Comorbid disease } \\
\hline Diabetes & $11(12.0)$ & $6(13.3)$ & $5(10.6)$ & 0.690 \\
\hline Hypertension & $31(33.7)$ & $21(46.7)$ & $10(21.3)$ & 0.010 \\
\hline \multicolumn{5}{|l|}{ Laboratory data } \\
\hline $\operatorname{eGFR}\left(\mathrm{mL} / \mathrm{min} / 1.73 \mathrm{~m}^{2}\right)$ & $13.2(8.1-25.5)$ & $8.6(7.0-13.2)$ & $24.0(12.9-40.2)$ & $<0.001$ \\
\hline Spot urine protein-to-creatinine ratio (g/g) & $2.2(1.5-3.9)$ & $2.1(1.5-4.1)$ & $2.3(1.5-3.8)$ & 0.645 \\
\hline Immunology ${ }^{a}$ & & & & 0.267 \\
\hline MPO-ANCA & $86(93.5)$ & 41 (91.1) & $45(95.7)$ & \\
\hline Normal glomeruli (\%) & $20.4(8.3-39.1)$ & $9.1(0.0-18.6)$ & $33.3(20.8-50.0)$ & $<0.001$ \\
\hline Crescentic glomeruli (\%) & $54.2(30.8-70.4)$ & $58.3(32.4-76.1)$ & $50.0(30.0-69.2)$ & 0.124 \\
\hline Globally sclerotic glomeruli (\%) & $14.8(0.0-30.2)$ & $23.3(6.3-38.8)$ & $8.3(0.0-23.1)$ & 0.004 \\
\hline $\mathrm{IF} / \mathrm{TA},>25 \%$ & $42(45.7)$ & $29(64.4)$ & $13(27.7)$ & $<0.001$ \\
\hline Histopathologic classification & & & & 0.003 \\
\hline Focal & $18(19.6)$ & $3(6.7)$ & 15 (31.9) & \\
\hline Crescentic & $30(32.6)$ & $14(22.2)$ & $16(34.0)$ & \\
\hline Mixed & $32(34.8)$ & $18(40.0)$ & $14(29.8)$ & \\
\hline Sclerotic & $12(13.0)$ & $10(22.2)$ & $2(4.3)$ & \\
\hline ANCA kidney risk score ${ }^{b}$ & $5.0(2.0-11.0)$ & $9.0(6.0-11.0)$ & $2.0(0-5.0)$ & $<0.001$ \\
\hline
\end{tabular}

Data are expressed as number only, mean \pm standard deviation, median (interquartile range), or number (\%).

ANCA, anti-neutrophil cytoplasmic antibody; eGFR, estimated glomerular filtration rate; ESKD, end-stage kidney disease; IF/TA, interstitial fibrosis/tubular atrophy; MPO, myeloperoxidase; PR3, proteinase 3.

${ }^{a}$ One patient (1 of $92,1.1 \%$ ) had positivity for both MPO- and PR3-ANCA.

${ }^{\mathrm{b}} \mathrm{S}$ um score of the clinicopathologic parameters (percentage of normal glomeruli, degree of IF/TA, and eGFR at the time of diagnosis).

glomeruli was 16.0, and the percentage of normal glomeruli was significantly lower $(\mathrm{p}<0.001)$ in the ESKD group. In contrast, the percentage of globally sclerotic glomeruli was significantly higher $(\mathrm{p}=0.004)$. A larger number of patients in the ESKD group had more than moderate $(>25 \%) \mathrm{IF} /$ TA compared to the non-ESKD group $(\mathrm{p}<0.001)$. In the histopathologic classification, the ESKD group had a higher rate of mixed and sclerotic classes, but the non-ESKD group had a higher rate of focal and crescentic classes (ESKD vs. non-ESKD; focal, $6.7 \%$ vs. $31.9 \%$; crescentic, $22.2 \%$ vs. $34.0 \%$; mixed, $40.0 \%$ vs. $29.8 \%$; and sclerotic, $22.2 \%$ vs. $4.3 \%$; $\mathrm{p}=$
0.003).

In the clinicopathologic classification, the sum of the ANCA kidney risk score was significantly higher in the ESKD group than in the non-ESKD group (9.0 [6.0-11.0] vs. 2.0 $[0.0-5.0] ; \mathrm{p}<0.001)$. There was a larger proportion of the high-risk group and a lower proportion of the low-risk group in the ESKD group than in the non-ESKD group (ESKD vs. non-ESKD; high, 28 [62.2\%] vs. 4 [8.5\%]; low, 2 [4.4\%] vs. 19 [40.4\%]; $\mathrm{p}<0.001)$. 
Table 2. Rate of progression to end-stage kidney disease according to the classifications

\begin{tabular}{|c|c|c|c|c|}
\hline Variable & 1 Year after diagnosis & p-value & Total follow-up period & p-value \\
\hline Histopathologic classification & & 0.003 & & 0.003 \\
\hline Focal & 2/18 (11.1) & & $3 / 18(16.7)$ & \\
\hline Crescentic & $10 / 30(33.3)$ & & $14 / 30(46.7)$ & \\
\hline Mixed & $15 / 32(46.9)$ & & $18 / 32(56.3)$ & \\
\hline Sclerotic & $9 / 12(75.0)$ & & $10 / 12(83.3)$ & \\
\hline Clinicopathologic classification & & $<0.001$ & & $<0.001$ \\
\hline Low & $1 / 21(4.8)$ & & $2 / 21(9.5)$ & \\
\hline Medium & $11 / 39(28.2)$ & & $15 / 39(38.5)$ & \\
\hline High & 24/32 (75.0) & & $28 / 32(87.5)$ & \\
\hline
\end{tabular}

Data are expressed as number (\%).
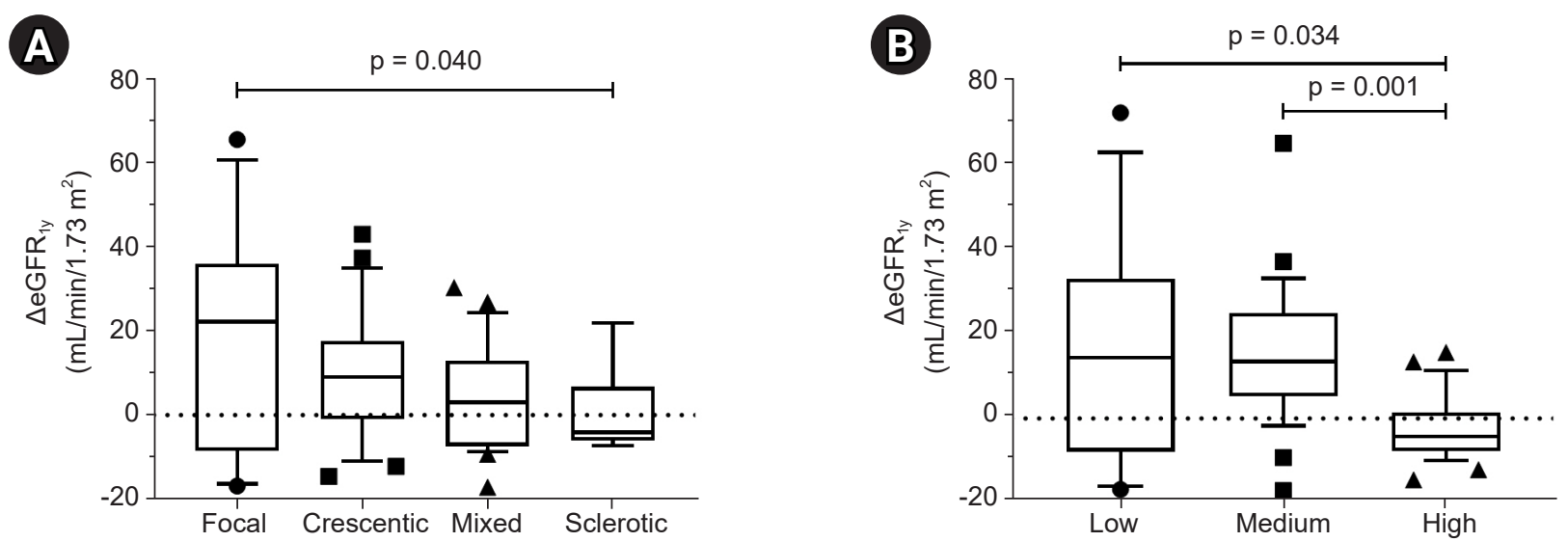

Figure 1. Change of eGFR during 1 year after diagnosis $\left(\Delta \mathrm{eGFR}_{1 \mathrm{y}}\right)$. (A) Comparison by histopathologic classification. (B) Comparison by clinicopathologic classification. Box plots indicate median and interquartile range. Whiskers indicate 10th and 90 th percentiles.

eGFR, estimated glomerular filtration rate.

\section{Renal outcomes and changes in renal function}

Thirty-six patients had progressed to ESKD at 1 year after diagnosis. The incidence of ESKD at 1 year was $11.1 \%$ (2 of 18 ), $33.3 \%$ ( 10 of 30 ), $46.9 \%$ ( 15 of 32 ), and $75.0 \%$ (9 of 12) in the focal, crescentic, mixed, and sclerotic classes, respectively $(\mathrm{p}=0.003)$ (Table 2$)$. The increasing trend with sequential category, focal, crescentic, mixed, and sclerotic classes, of the proportion of patients who developed ESKD, was also maintained during the total follow-up period ( $16.7 \%$ [ 3 of 18], $46.7 \%$ [14 of 30], 56.3\% [18 of 32], and 83.3\% [10 of 12], respectively; $p=0.003$ ). In the clinicopathologic classification, the incidence of ESKD increased in the order of the low-, medium-, and high-risk groups both at 1 year after diagnosis and the entire follow-up period ( 1 year: $4.8 \%$ [1 of 21], $28.2 \%$ [11 of 39], and $75.0 \%$ [24 of 32]; total followup: $9.5 \%$ [ 2 of 21 ], $38.5 \%$ [ 15 of 39], and $87.5 \%$ [ 28 of 32], respectively; both $\mathrm{p}<0.001$ ).

As an index of therapeutic response, the changes in eGFR at 1 year after diagnosis $\left(\triangle \mathrm{eGFR}_{\mathrm{ly}}\right)$ were compared (Fig. 1$)$. In the histopathologic classification, $\Delta \mathrm{eGFR}_{\mathrm{ly}}$ improved in the order of focal, crescentic, mixed, and sclerotic classes (Fig. 1A). $\Delta \mathrm{eGFR}_{\mathrm{yy}}$ was significantly higher in the focal class than in the sclerotic class $(p=0.040)$. In the clinicopathologic classification, the high-risk group had worse $\Delta \mathrm{eGFR}_{\mathrm{ly}}$ than both the low- and medium-risk groups ( $\mathrm{p}=0.034$ and $\mathrm{p}=$ 0.001, respectively) (Fig. 1B). In the multiple linear regression analyses for $\triangle \mathrm{eGFR}_{\mathrm{ly}}$, the mixed and sclerotic classes showed a faster decline of eGFR than the focal class after adjusting for age, sex, diabetes, and hypertension (model 1 with the histopathologic classification; both $\mathrm{p}<0.05$ ) (Table 3). After adjusting for the same factors, the decline of eGFR was greater in the high-risk group than in the low-risk group (model 2 with the clinicopathologic classification; $\mathrm{p}=0.008$ ), 
Table 3. Linear regression analysis of the change in eGFR during 1-year after diagnosis

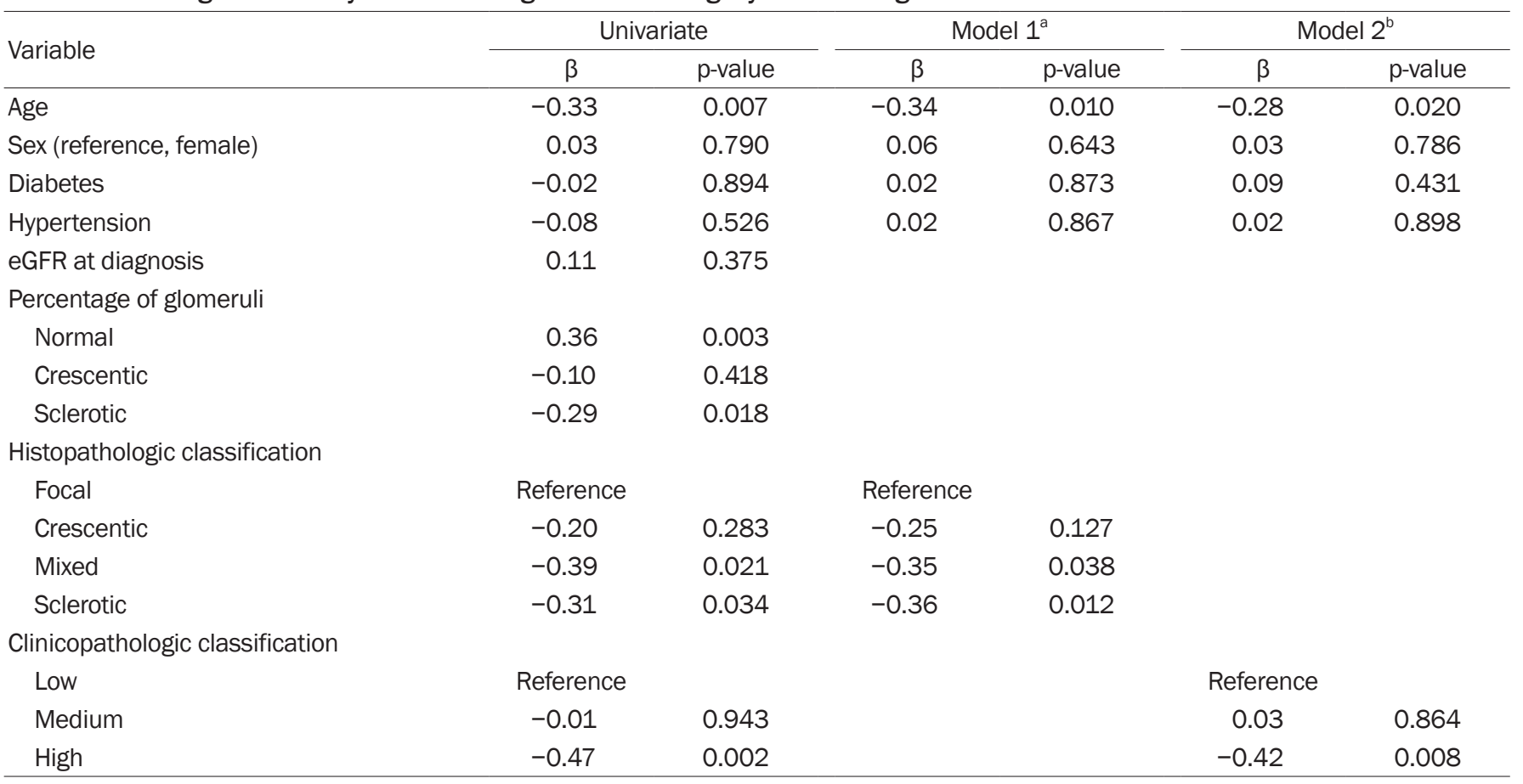

eGFR, estimated glomerular filtration rate.

${ }^{a}$ Model 1 for histopathologic classification: adjusted for age, sex, diabetes, and hypertension. ${ }^{b}$ Model 2 for clinicopathologic classification: adjusted for age, sex, diabetes, and hypertension.

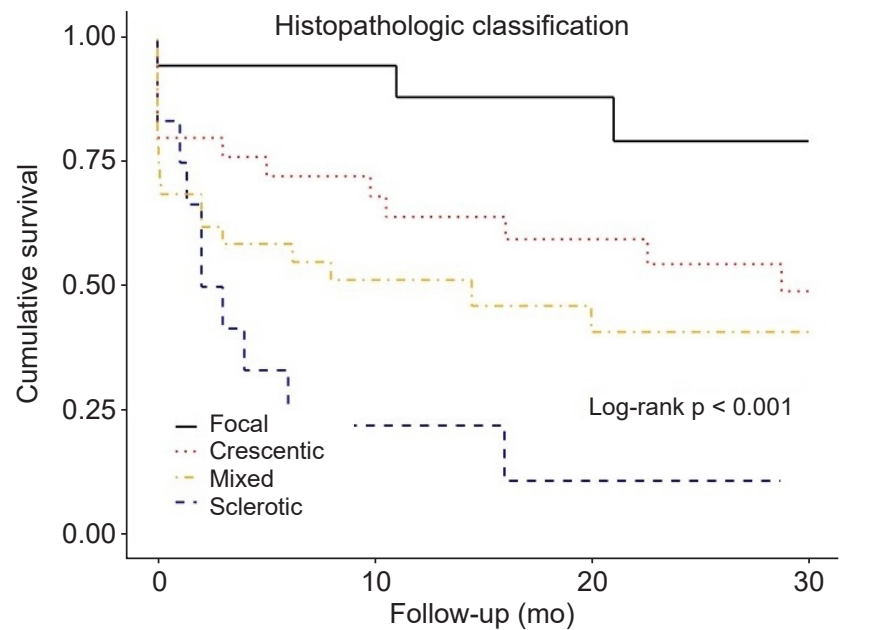

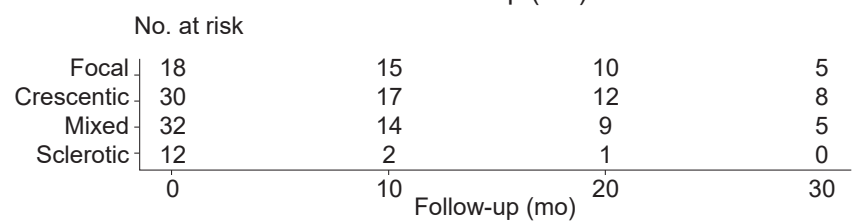

Figure 2. Kaplan-Meier curve for renal survival according to histopathologic classification.

but the medium-risk group did not show significance. Aging was identified as a negative predictor for $\Delta \mathrm{eGFR}_{\mathrm{ly}}$ in both models $(\mathrm{p}<0.05)$.

\section{Prediction of renal survival}

In the Kaplan-Meier analysis, renal survival was significantly different according to the histopathologic classification (logrank $\mathrm{p}<0.001$ ) (Fig. 2). The mixed and sclerotic classes had worse renal outcomes than the focal class $(\mathrm{p}=0.008$ and $\mathrm{p}$ $<0.001$, respectively). The crescentic class had a tendency for poorer renal survival than the focal class $(p=0.059)$. When dividing patients according to the cutoff of each parameter in the clinicopathologic classification, all three items (percentage of normal glomeruli, IF/TA, and baseline eGFR) were individually associated with renal survival (all $\log$-rank $\mathrm{p}<0.001$ ), but normal glomeruli ratios of $>25 \%$ and $10 \%$ to $25 \%$ did not differ in renal survival $(\mathrm{p}>0.05$; Fig. $3 \mathrm{~A}-$ C). In the clinicopathologic classification, renal survival was decreased in the a low, medium, and high order of risk (log$\operatorname{rankp}<0.001$ ) (Fig. 3D).

In the multivariate Cox regression analysis of renal outcome, the mixed and sclerotic classes were independent predictors for ESKD (vs. the focal class; mixed class: 

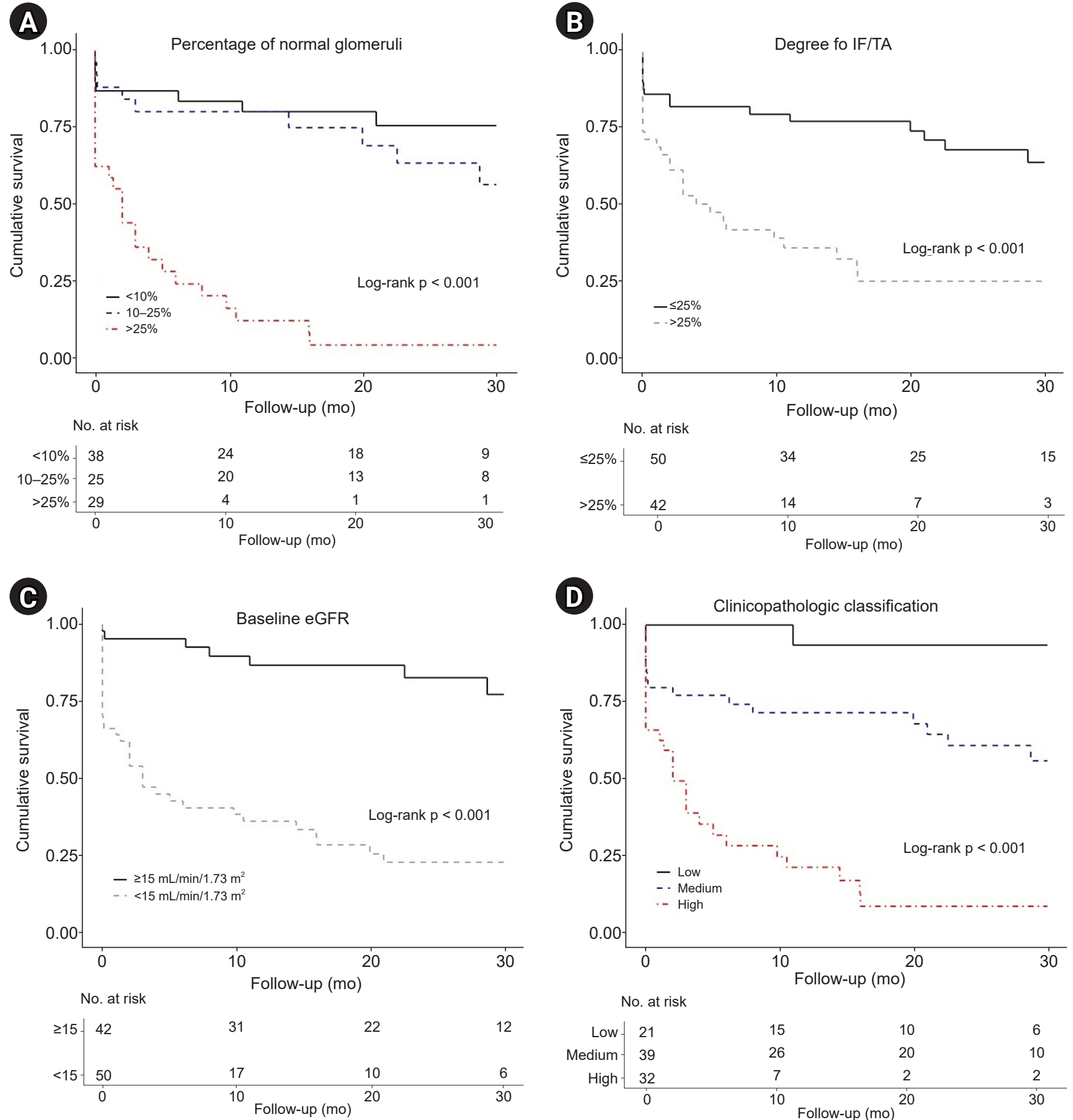

No. at risk

\begin{tabular}{|c|c|c|c|c|}
\hline Low & 21 & 15 & 10 & 6 \\
\hline Medium & 39 & 26 & 20 & 10 \\
\hline High & 32 & 7 & 2 & 2 \\
\hline & 0 & 10 & 20 & 30 \\
\hline
\end{tabular}

Figure 3. Kaplan-Meier curve for renal survival according to clinicopathologic classification and each included parameter. (A) Percentage of normal glomeruli. (B) Degree of interstitial fibrosis/tubular atrophy. (C) Estimated glomerular filtration rate (eGFR) at the time of diagnosis. (D) Clinicopathologic classification combining each parameter.

adjusted hazard ratio [aHR], 4.23; 95\% confidence interval [CI], 1.23-14.58; $\mathrm{p}=0.022$; sclerotic class: aHR, 5.05; 95\% CI, 1.32-19.31; $\mathrm{p}=0.018$; model 1) (Table 4). The high-risk group of the clinicopathologic classification was independently associated with the worse renal outcome (vs. low-risk; aHR, 6.56; 95\% CI, 1.25-34.26; $\mathrm{p}=0.026$ ), but the medium-risk 
Table 4. Predictors for progression to end-stage kidney disease in the Cox proportional hazards model

\begin{tabular}{|c|c|c|c|c|c|c|}
\hline \multirow{2}{*}{ Variable } & \multicolumn{2}{|l|}{ Univariate } & \multicolumn{2}{|l|}{ Model $1^{a}$} & \multicolumn{2}{|l|}{ Model $2^{b}$} \\
\hline & $\mathrm{HR}(95 \% \mathrm{Cl})$ & p-value & aHR $(95 \% \mathrm{Cl})$ & p-value & aHR (95\% Cl) & p-value \\
\hline Age & $1.03(1.01-1.06)$ & 0.015 & $1.02(0.99-1.04)$ & 0.210 & $1.01(0.99-1.04)$ & 0.263 \\
\hline Sex & $1.22(0.67-2.20)$ & 0.514 & $1.23(0.66-2.28)$ & 0.520 & $1.32(0.72-2.40)$ & 0.372 \\
\hline Diabetes & $1.42(0.60-3.37)$ & 0.425 & & & & \\
\hline Hypertension & 1.85 (1.03-3.33) & 0.039 & $0.95(0.50-1.79)$ & 0.871 & $1.15(0.61-2.17)$ & 0.674 \\
\hline eGFR at diagnosis & $0.90(0.86-0.95)$ & $<0.001$ & $0.91(0.87-0.96)$ & $<0.001$ & $0.95(0.91-1.00)$ & 0.049 \\
\hline \multicolumn{7}{|c|}{ Percentage of glomeruli } \\
\hline Normal & $0.96(0.94-0.98)$ & $<0.001$ & & & & \\
\hline Crescentic & $1.01(1.00-1.02)$ & 0.156 & & & & \\
\hline Sclerotic & $1.02(1.01-1.04)$ & $<0.001$ & & & & \\
\hline \multicolumn{7}{|c|}{ Histopathologic classification } \\
\hline Focal & Reference & & Reference & & & \\
\hline Crescentic & $3.40(0.98-11.85)$ & 0.054 & $2.45(0.69-8.67)$ & 0.165 & & \\
\hline Mixed & $5.04(1.48-17.19)$ & 0.010 & $4.23(1.23-14.58)$ & 0.022 & & \\
\hline Sclerotic & $9.88(2.66-36.62)$ & 0.001 & $5.05(1.32-19.31)$ & 0.018 & & \\
\hline \multicolumn{7}{|c|}{ Clinicopathologic classification } \\
\hline Low & Reference & & & & Reference & \\
\hline Medium & $4.40(1.01-19.23)$ & 0.049 & & & $2.56(0.54-12.12)$ & 0.237 \\
\hline High & $18.63(4.38-79.17)$ & $<0.001$ & & & $6.56(1.25-34.26)$ & 0.026 \\
\hline
\end{tabular}

aHR, adjusted hazard ratio; $\mathrm{Cl}$, confidence interval; eGFR, estimated glomerular filtration rate; HR, hazard ratio.

${ }^{a}$ Model 1 for histopathologic classification: adjusted for age, sex, hypertension, and eGFR at diagnosis. ${ }^{\mathrm{b}}$ Model 2 for clinicopathologic classification: adjusted for age, sex, hypertension, and eGFR at diagnosis.

group was not associated with poor renal outcome compared to the low-risk group (model 2).

In addition, the associations of active inflammatory lesions in histopathology and renal outcomes are shown in Supplementary Table 1 (available online). Among the active inflammatory lesions, such as glomerular fibrinoid necrosis, peritubular capillaritis, tubulitis, and interstitial inflammation, moderate to severe interstitial inflammation was an independent predictor for ESKD after adjustment of age, sex, and hypertension (aHR, 2.40; 95\% CI, 1.09-5.31; p = $0.031)$.

\section{Discussion}

This study evaluated the renal prognostic value of both histopathologic and clinicopathologic classifications among Korean AAGN patients. Unlike in western countries [9,10,18], the Korean AAGN cohort had a higher proportion of MPOANCA positive and higher incidence of progression to ESKD. Previous studies have shown that patients with MPOANCA have more abundant active and chronic lesions in renal histopathology than those of proteinase 3-ANCA positive patients. Therefore, MPO-ANCA positive patients have a higher risk of renal failure [19-21]. In the histopathologic classification according to glomerular pathology, the focal class had the best renal survival, and the mixed and sclerotic classes had the worse and worst renal survival, respectively. However, the crescentic class did not differ in renal survival compared to the focal class. In the clinicopathologic classification according to the percentage of normal glomeruli, degree of IF/TA, and baseline renal function, the high-risk group had poor renal survival than the low- or medium-risk group; however, there was no difference in renal survival between the low- and medium-risk groups. These results demonstrated that the classifications that originated from western countries were moderate in predicting renal outcomes in Asian AAGN patients. In particular, they showed high predictability for renal outcomes in the worst prognosis groups, such as the sclerotic class or the high-risk group.

AAGN is the most common manifestation of AAV and is highly associated with morbidity and mortality [6,20]. There are several known prognostic factors for progression to ESKD in AAGN, but it is difficult to predict renal outcomes 
accurately with any single factor $[5,7,10]$. Berden et al. [9] developed a simple histopathologic classification from European AAGN patients, which focused on the glomerular findings, except background tubulointerstitial findings and clinical findings. They reported that the focal class had a favorable outcome, the mixed class had an intermediate outcome, and the sclerotic class had the highest risk of not recovering renal function and progression to ESKD. Several western and Asian studies have also evaluated the prognostic value of the histopathologic classification $[6,12,20,22,23]$. Most studies, including a recent metaanalysis, showed similar outcomes: the best outcome in the focal class and the worst outcome in the sclerotic class $[6,12,20,22,23]$. Globally sclerotic glomeruli are not AAGNspecific findings. However, regardless of the AAGN disease activity, these chronic lesions indicate less response to treatment and a higher risk of progression to $\operatorname{ESKD}[7,24]$. Unlike these classes, there is a controversy about the outcome of the crescentic and mixed classes. Some studies have reported that the crescentic class has a worse outcome than the focal or mixed class $[6,23,25]$. Others have reported a non-inferior outcome of the crescentic class compared to the focal class because of a good therapeutic response $[9,12,20]$. The results of this study are consistent with the latter-with Berden et al. [9] and the Japanese cohort studies [12]. Among Korean AAGN patients, the outcome of the crescentic class was relatively good and not inferior to the focal class. Recently, van Daalen et al. [26] reported a validation study for AAGN from patients worldwide. The $\triangle \mathrm{eGFR}$ ly was significantly higher in the crescentic class than in the mixed class despite similar renal function at the time of diagnosis, indicating that the crescentic class had a better treatment response. They explained that this would be related to the different composition of crescent typesthat the crescentic class is characterized by a majority of cellular crescents and these are reversible lesions, but the mixed class has more heterogeneous crescent types, such as fibrocellular and fibrous crescents, with poor response to treatment $[26,27]$. These results may also be related to the association of different human leukocyte antigen by ethnicity in AAV patients [11]. Thus, based on the results of this study, Korean AAGN patients with the focal or crescentic class will need active treatment.

Brix et al. [10] developed a more complex classification using a calculation based on the ANCA kidney risk score. All three factors included in this classification (normal glomeruli ratio, degree of IF/TA, and baseline renal function) are well- established prognostic markers of AAGN in previous studies $[4,5,7,15,24]$. They determined the appropriate cutoff through rigorous analysis of the German AAGN cohort and divided the groups by scoring. The advantage of this classification is that it reflects both clinical laboratory (eGFR) and histopathologic (normal glomeruli and IF/TA) parameters. However, although these clinicopathologic variables were aggregated, the renal prognosis of the lowand medium-risk groups did not differ in the Korean cohort. A recent Turkish study also reported the same results as in this study [28]. There are some possible reasons for this inconsistent result. First, the cutoff value of the percentage of normal glomeruli was too low. Berden et al. [9] considered significance when the normal glomeruli percentage was higher than $50 \%$, but Brix et al. [10] set the cutoffs at a lower rate of $10 \%$ and $25 \%$. Hilhorst et al. [25] reported the worst survival in AAGN patients with normal glomeruli percentage of $<25 \%$. Tanna et al. [5] reported a significant difference in renal survival among normal glomeruli percentages of $0 \%$ to $20 \%, 21 \%$ to $50 \%$, and $>51 \%$. There was no difference in renal survival between normal glomeruli percentages of $10 \%$ to $25 \%$ and $>25 \%$ in the results of this study. Therefore, a modified cutoff of the normal glomeruli ratio is required to accurately predict survival among Korean AAGN patients. Second, the proportion of patients who progressed to ESKD was much higher in this study. The cohort in this study had a lower baseline eGFR and a higher percentage of sclerotic glomeruli than the cohort studied by Brix et al. [10], and the proportion of MPO-ANCA positive was also higher. All these indicators suggest a higher risk of progression to ESKD. Additionally, ethnicity may have possibly contributed to the difference in results from the previous study.

In addition to the aforementioned histopathologic classification, an analysis of the association between histopathologic findings reflecting active lesions and renal survival revealed that moderate to severe interstitial inflammation independently increased the risk of ESKD. This result is consistent with a previous study that reported diffuse interstitial infiltration of inflammatory cells is a predictor for poor renal outcome in European patients with AAGN [4].

The incidence of AAGN increases with age. It is the second most common glomerulonephritis among Korean 
patients aged above 70 years who underwent renal biopsy $[29,30]$. AAGN patients have a high risk of not only renal failure but also death, so prompt diagnosis and proper management are necessary. However, the mortality of AAV patients within the first 12 months after diagnosis was as high as $11 \%$, and half of them died from infection [31]. Intensive immunosuppression also results in other adverse events, such as malignancy, bone marrow suppression, and gastrointestinal bleeding [31]. Thus, especially in elderly patients who are vulnerable to the adverse effects of immunosuppressive therapy, it is important to accurately predict the therapeutic response and provide an appropriate intensity of treatment. Neither the histopathologic nor the clinicopathologic classification perfectly predicts renal outcomes in Korean AAGN patients. However, both classifications showed high predictability of ESKD progression for patients in either the sclerotic class or the high-risk group. In these patients, treatment intensity should be adjusted to consider the worse renal prognosis with less therapeutic response. Also, background histopathologic findings such as interstitial inflammation need to be considered more to accurately predict the renal outcome. In a recently published study on crescentic glomerulonephritis patients, severe arteriosclerosis and tertiary lymphoid organ formation are independent predictors for renal survival after adjusting for age and baseline renal function [14]. By considering indicators that reflect chronic changes and inflammation activity, a more accurate prediction of renal outcomes will be possible.

The strength of this study is that it analyzed unselected patients who were diagnosed with AAGN at two universitybased hospitals in Korea. For the first time in Korean AAGN patients, the predictive value of two European origin classifications was assessed. However, there are several limitations. First, this is a retrospective study, so there is a limitation to documenting the effectiveness of various treatments on renal outcomes. Second, the number of study patients was relatively small, although patients diagnosed with AAGN for a long period were included. This may reduce the validity. However, in both classifications, the number of patients was distributed appropriately in each class. Third, patients were treated at the discretion of the clinicians at each hospital. However, although there was no standard treatment, the Korean national health insurance system covers most medical expenses; therefore, treatment is performed according to the same national health insurance guidelines. Fourth, there is a possibility of sample bias. Partial tissue specimen cannot reflect the overall renal disease condition. However, like other AAGN studies $[5,8]$, the bias was reduced by screening specimens that contained more than 10 glomeruli.

In conclusion, both histopathologic and clinicopathologic classifications were moderately able to predict the renal prognosis of Korean AAGN patients. For the sclerotic class or the high-risk group at diagnosis, the predictability for poor renal outcome was high due to poor response to treatment, and they had a high risk of progression to ESKD. Accordingly, clinicians need to balance the risks and benefits of treatment in Korean AAGN patients by identifying the group at high risk for renal outcomes.

\section{Conflicts of interest}

All authors have no conflicts of interest to declare.

\section{Authors' contributions}

Conceptualization: JHL, SHP

Data curation: JHL, YJ, MHH, YJK

Investigation: JHL, HYJ, JYC, JHC, CDK, YLK, HL, DKK, KCM

Project administration: SHP

Writing-original draft: JHL, SHP

Writing-review \& editing: All authors

All authors read and approved the final manuscript.

\section{ORCID}

Jeong-Hoon Lim, https://orcid.org/0000-0001-5517-9886

Man-Hoon Han, https://orcid.org/0000-0001-8856-553X Yong-Jin Kim, https://orcid.org/0000-0002-9867-0752 Yena Jeon, https://orcid.org/0000-0002-4857-8616 Hee-Yeon Jung, https://orcid.org/0000-0003-0232-7202 Ji-Young Choi, https://orcid.org/0000-0002-9774-3665 Jang-Hee Cho, https://orcid.org/0000-0002-7031-5214 Chan-Duck Kim, https://orcid.org/0000-0002-4648-0324 Yong-Lim Kim, https://orcid.org/0000-0002-1344-3455 Hajeong Lee, https://orcid.org/0000-0002-1873-1587 Dong Ki Kim, https://orcid.org/0000-0002-5195-7852 Kyung Chul Moon, https://orcid.org/0000-0002-1969-8360 Sun-Hee Park, https://orcid.org/0000-0002-0953-3343 


\section{References}

1. Jennette JC, Falk RJ, Hu P, Xiao H. Pathogenesis of antineutrophil cytoplasmic autoantibody-associated small-vessel vasculitis. Annu Rev Pathol 2013;8:139-160.

2. Jennette JC, Falk RJ, Bacon PA, et al. 2012 revised International Chapel Hill Consensus Conference Nomenclature of Vasculitides. Arthritis Rheum 2013;65:1-11.

3. Pettersson EE, Sundelin B, Heigl Z. Incidence and outcome of pauci-immune necrotizing and crescentic glomerulonephritis in adults. Clin Nephrol 1995;43:141-149.

4. Bajema IM, Hagen EC, Hermans J, et al. Kidney biopsy as a predictor for renal outcome in ANCA-associated necrotizing glomerulonephritis. Kidney Int 1999;56:1751-1758.

5. Tanna A, Guarino L, Tam FW, et al. Long-term outcome of antineutrophil cytoplasm antibody-associated glomerulonephritis: evaluation of the international histological classification and other prognostic factors. Nephrol Dial Transplant 2015;30:11851192.

6. Ellis CL, Manno RL, Havill JP, Racusen LC, Geetha D. Validation of the new classification of pauci-immune glomerulonephritis in a United States cohort and its correlation with renal outcome. BMC Nephrol 2013;14:210.

7. de Lind van Wijngaarden RA, Hauer HA, Wolterbeek R, et al. Clinical and histologic determinants of renal outcome in ANCAassociated vasculitis: a prospective analysis of 100 patients with severe renal involvement. J Am Soc Nephrol 2006;17:2264-2274.

8. Berden AE, Jones RB, Erasmus DD, et al. Tubular lesions predict renal outcome in antineutrophil cytoplasmic antibodyassociated glomerulonephritis after rituximab therapy. J Am Soc Nephrol 2012;23:313-321.

9. Berden AE, Ferrario F, Hagen EC, et al. Histopathologic classification of ANCA-associated glomerulonephritis. J Am Soc Nephrol 2010; 21:1628-1636.

10. Brix SR, Noriega M, Tennstedt P, et al. Development and validation of a renal risk score in ANCA-associated glomerulonephritis. Kidney Int 2018;94:1177-1188.

11. Chang DY, Luo H, Zhou XJ, Chen M, Zhao MH. Association of HLA genes with clinical outcomes of ANCA-associated vasculitis. Clin J Am Soc Nephrol 2012;7:1293-1299.

12. Iwakiri T, Fujimoto S, Kitagawa K, et al. Validation of a newly proposed histopathological classification in Japanese patients with anti-neutrophil cytoplasmic antibody-associated glomerulonephritis. BMC Nephrol 2013;14:125.

13. Levey AS, Stevens LA. Estimating GFR using the CKD Epidemiology
Collaboration (CKD-EPI) creatinine equation: more accurate GFR estimates, lower CKD prevalence estimates, and better risk predictions. Am J Kidney Dis 2010;55:622-627.

14. Lim JH, Han MH, Kim YJ, et al. Novel histopathologic predictors for renal outcomes in crescentic glomerulonephritis. PLoS One 2020;15:e0236051.

15. Bajema IM, Hagen EC, Hansen BE, et al. The renal histopathology in systemic vasculitis: an international survey study of inter- and intra-observer agreement. Nephrol Dial Transplant 1996;11:19891995.

16. Jennette JC. Rapidly progressive crescentic glomerulonephritis. Kidney Int 2003;63:1164-1177.

17. Radhakrishnan J, Cattran DC. The KDIGO practice guideline on glomerulonephritis: reading between the (guide)lines: application to the individual patient. Kidney Int 2012;82:840856.

18. Bjørneklett R, Sriskandarajah S, Bostad L. Prognostic value of histologic classification of ANCA-associated glomerulonephritis. Clin J Am Soc Nephrol 2016;11:2159-2167.

19.Hauer HA, Bajema IM, van Houwelingen HC, et al. Renal histology in ANCA-associated vasculitis: differences between diagnostic and serologic subgroups. Kidney Int 2002;61:80-89.

20. Chen YX, Xu J, Pan XX, et al. Histopathological classification and renal outcome in patients with antineutrophil cytoplasmic antibodies-associated renal vasculitis: a study of 186 patients and metaanalysis. J Rheumatol 2017;44:304-313.

21. Cornec D, Cornec-Le Gall E, Fervenza FC, Specks U. ANCAassociated vasculitis: clinical utility of using ANCA specificity to classify patients. Nat Rev Rheumatol 2016;12:570-579.

22. Noone DG, Twilt M, Hayes WN, et al. The new histopathologic classification of ANCA-associated GN and its association with renal outcomes in childhood. Clin J Am Soc Nephrol 2014;9:16841691.

23. Chang DY, Wu LH, Liu G, Chen M, Kallenberg CG, Zhao MH. Re-evaluation of the histopathologic classification of ANCAassociated glomerulonephritis: a study of 121 patients in a single center. Nephrol Dial Transplant 2012;27:2343-2349.

24. Hauer HA, Bajema IM, Van Houwelingen HC, et al. Determinants of outcome in ANCA-associated glomerulonephritis: a prospective clinico-histopathological analysis of 96 patients. Kidney Int 2002;62:1732-1742.

25. Hilhorst M, Wilde B, van Breda Vriesman P, van Paassen P, Cohen Tervaert JW; Limburg Renal Registry. Estimating renal survival using the ANCA-associated GN classification. J Am Soc Nephrol 2013;24:1371-1375. 
26. van Daalen EE, Wester Trejo MAC, Göçeroğlu A, et al. Developments in the histopathological classification of ANCA-associated glomerulonephritis. Clin J Am Soc Nephrol 2020;15:1103-1111.

27. Atkins RC, Nikolic-Paterson DJ, Song Q, Lan HY. Modulators of crescentic glomerulonephritis. J Am Soc Nephrol 1996;7:22712278.

28. Gercik O, Bilgin E, Solmaz D, et al. Histopathological subgrouping versus renal risk score for the prediction of end-stage renal disease in ANCA-associated vasculitis. Ann Rheum Dis 2020;79:675-676.
29. Yim T, Kim SU, Park S, et al. Patterns in renal diseases diagnosed by kidney biopsy: a single-center experience. Kidney Res Clin Pract 2020;39:60-69.

30. Bomback AS, Appel GB, Radhakrishnan J, et al. ANCA-associated glomerulonephritis in the very elderly. Kidney Int 2011;79:757764.

31. Little MA, Nightingale P, Verburgh CA, et al. Early mortality in systemic vasculitis: relative contribution of adverse events and active vasculitis. Ann Rheum Dis 2010;69:1036-1043. 